\title{
Outcomes and dynamic changes of the mitral valve after surgical repair of mitral regurgitation in patients with an atrial septal defect
}

\author{
Ok Jeong Lee ${ }^{1}$, Jinyoung Song ${ }^{2}$, June Huh ${ }^{2}$, I-Seok Kang ${ }^{2}$, Ji-Hyuk Yang ${ }^{1}$, \\ Tae-Gook Jun ${ }^{1}$ \\ ${ }^{1}$ Department of Thoracic and Cardiovascular Surgery, Samsung Medical Center, Sungkyunkwan University School of Medicine, \\ Seoul, Korea \\ ${ }^{2}$ Department of Pediatrics, Samsung Medical Center, Sungkyunkwan University School of Medicine, Seoul, Korea
}

Received: April 26, 2019

Revised: July 15, 2019

Accepted: July 16, 2019

Corresponding author:

Jinyoung Song

Department of Pediatrics,

Samsung Medical Center,

Sungkyunkwan University

School of Medicine, 81 Irwon-

ro, Gangnam-gu, Seoul 06351,

Korea

Tel: +82-2-3410-3539

E-mail: amyjys@skku.edu

\section{ABSTRACT}

Purpose: We purposed to determine the outcomes of surgical mitral valve repair in patients with atrial septal defect (ASD) and mitral regurgitation (MR) and determine factors associated with good outcomes.

Methods: Sixty-one patients after operation for ASD and MR were enrolled. Mitral valve annular diameter, lengths of mitral leaflets, and motion angles of both leaflets were measured on echocardiographic images.

Results: The severity of MR and mitral valve prolapse improved after surgery from 2.3 \pm 0.7 to $1.7 \pm 0.9$ and from $41.8 \%$ to $16.4 \%$, respectively. In patients with postoperative MR grade $<3$, angles of both leaflets on systole and motion angles of the posterior leaflet were changed. Operation at an earlier age $(28.0 \pm 18.9$ years vs. $60.3 \pm 10.1$ years $)$ and chordae repair $(70.2 \%$ vs. $25.0 \%)$ were performed more frequently in patients with postoperative MR grade $<3$. More MAZE operations were performed in patients with postoperative MR grade $\geq 3$ ( $21.3 \%$ vs. $87.5 \%)$.

Conclusion: Operation age, MAZE operation, and chordae repair affected postoperative MR. Not only prolapse, but also the angles of both leaflets on systole and the motion angle of PML were significantly improved in patients with postoperative MR grade $<3$.

Keywords: Heart septal defects, atrial; Mitral valve insufficiency; Mitral valve repair
This is an Open Access article distributed under the terms of the Creative Commons Attribution Non-Commercial License (http:// creativecommons.org/licenses/ by-nc/4.0/).

\section{INTRODUCTION}

Atrial septal defect (ASD) is a common congenital heart disease encountered in adult patients [1] and a well-known congenital cardiac anomaly that results in chronic right heart volume overload and left ventricular volume depletion [2]. Mitral regurgitation (MR) has been discovered in less than $10 \%$ of adult patients with ASD [3-5]. On the one hand, ASD closure might induce right ventricular remodeling that restores abnormal left ventricular geometry, thus im- 
proving MR [6,7]. Therefore, isolated surgical ASD closure or transcatheter ASD closure might be sufficient to correct MR associated with ASD [6-8]. On the other hand, aggravation or new onset of MR after ASD closure has been reported [9-13]; therefore, concomitant surgical MR repair and ASD closure rather than transcatheter closure of ASD should be considered in high-risk patients. However, to the best of our knowledge, outcomes of surgical repair of MR in patients with ASD have not been reported. Our aims in this study were therefore to evaluate surgical outcomes and determine the factors that influence postoperative MR in ASD patients.

\section{METHODS}

The medical records of 73 consecutive patients who simultaneously underwent surgical closure of isolated secundum ASD and repair of MR at Samsung Medical Center between January 2005 and December 2014 were reviewed retrospectively. Eighteen patients with MR suspiciously associated with rheumatic carditis or whose medical data were inadequate or absent were excluded. This study was approved with waiver of informed consent by the Ethics Committee of Samsung Medical Center (IRB: 2017-04-047).

\section{Operative procedures}

Surgical approach was decided by multi-disciplinary discussion of experts including pediatric cardiologists and pediatric cardiac surgeons. Surgical repair was accepted when the degree of MR was more than grade 2 and MR was not expected to regress spontaneously. Direct closure or patch closure was used for ASD closure. Procedures for surgical repair for MR were chosen based on the surgeon's preference after cautious visual inspection of the mitral valve (MV) in the operation room and were reviewed on the surgical record. The most common procedure was annuloplasty, including ring annuloplasty, which was performed in 49 patients (89.1\%). Leaflet repair, including commissuroplasty and leaflet plication, was performed in 14 patients (25.5\%). Chordae repair was done in 35 patients (63.6\%). The MAZE operation was performed concomitantly for significant atrial fibrillation in 17 patients (30.9\%).

\section{Echocardiography}

All patients with ASD who were scheduled for surgical correction underwent two-dimensional and Doppler transthoracic echocardiography before and after surgery. Echocardiography was performed according to the American Society of Echocardiography guidelines [14] with available machines, namely a SIEMENS X-300 (Siemens, Mountain View, CA, USA), ACUSON Sequoia C512 (Siemens), or VIVID E9 (General Electric Medical Systems, Milwaukee, WI, USA). The last echocardiographic images after surgery except within the immediate postoperative period were selected to represent postoperative status. The median period of last echocardiographic evaluation from operation was 15.0 months. MV annulus size, motion, and sizes of both leaflets were evaluated. Measurements were performed retrospectively from stored echocardiographic images in one session by a single author (J.S.), and the mean value was calculated from three measurements. Severity of MR was assessed visually by the spatial distribution of color flow. Degrees of MR were defined as follows: 1 , minimal; 2 , mild; 3 , moderate; and 4 , severe.

\section{Diameter of the mitral valve annulus and sizes of leaflets}

The diameter of the MV annulus was measured in the apical four-chamber image during systole, and the largest diameter was selected. Lengths of the anterior and posterior MV leaf-
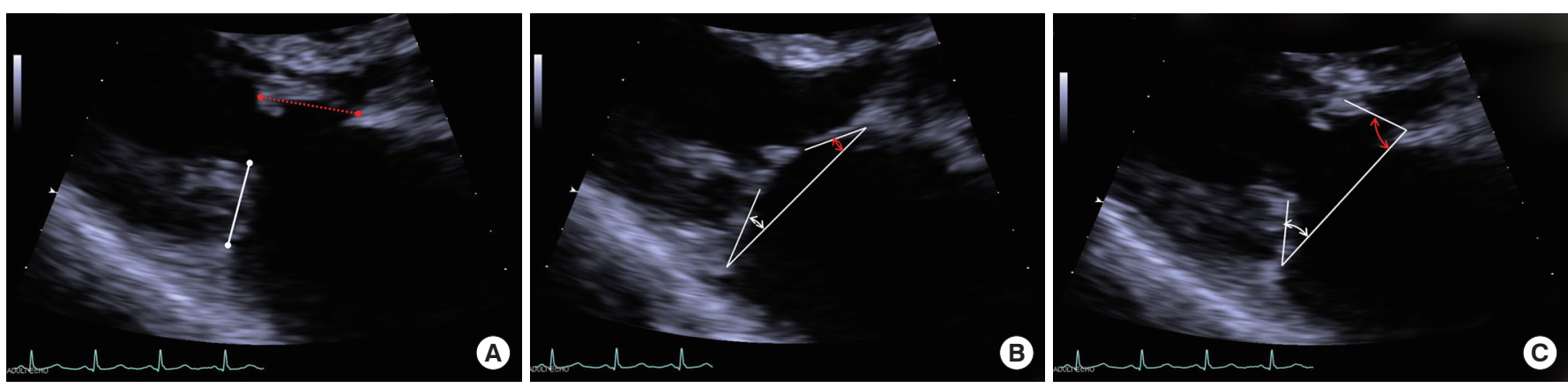

Fig. 1. (A) Measurement of the lengths of the anterior and posterior mitral valve leaflets; length between the tip of the leaflet and hinge point of the leaflet that attached to the mitral annulus. Measurement of the angles of both leaflets; (B) closed angles of both mitral leaflets at end-systole and $(C)$ open angle of both mitral leaflets at end-diastole based on the mitral annulus line. 


\section{PRECISION AND FUTURE MIEDICINE}

Outcomes after MR repair in ASD patients

lets were measured between the tip of the leaflet and hinge point of leaflet that attached to the mitral annulus (Fig. 1A).

\section{Leaflet motion}

Angles of the anterior mitral leaflet (AML) and posterior mitral leaflet (PML) from the MV annulus line were measured at both end-systole and end-diastole (Fig. 1B, C). Difference between the angles at end-diastole and end-systole was defined as the motion angle of the leaflet. Leaflet prolapse was diagnosed when the angle had a negative value, and mitral valve prolapse (MVP) was diagnosed when at least one of the two leaflets was prolapsed (Fig. 2). And then the mechanism of MR was analyzed based on the motion of each mitral leaflet.

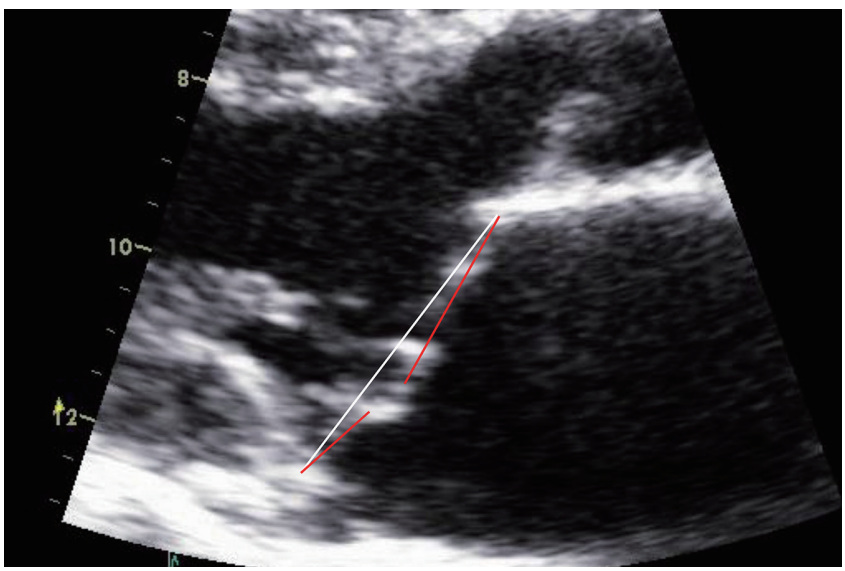

Fig. 2. Demonstration of measurement of the angles of both mitral leaflets on echocardiography in patient with anterior and posterior mitral valve prolapse $\left(-15.3^{\circ}\right.$ and $\left.-12.5^{\circ}\right)$.

\section{Statistical analysis}

All continuous data were described as means and standard deviations. Values were compared between groups using Student's t-test and the paired t-test. Wilcoxon signed rank test was used for nonparametric data and the McNemar test for categorical data. All statistical analyses were performed using the SPSS statistical software program for Windows version 23.0 (IBM Co., Armonk, NY, USA), and a P-valve less than 0.05 was considered statistically significant.

\section{RESULTS}

\section{Preoperative mitral valve}

No immediate or late mortality was observed during the follow-up period. The median age of patients at the operation was 34.0 years, and 33 patients (60.0\%) were female. Preoperative MR grade $\geq 3$ and MVP were observed in 13 patients and 23 patients, respectively. Other data are presented in Table 1. Patients with more than a moderate degree of preoperative MR did not have any variables that were significantly different from those of other patients; although MVP was more frequent in the former patient group, this was not statistically significant (61.5\% vs. $35.7 \%, P=0.093)$ (Table 2 ).

\section{Changes after repair}

Table 1 shows significant changes in degree of MR, MVP, leaflet angles on systole, and motion angles of both leaflets after surgery. Angles of both leaflets on systole were larger after surgery and motion angles were smaller, which could explain

Table 1. Post-operative changes

\begin{tabular}{|c|c|c|c|}
\hline Variable & Preoperative & Postoperative & P-value ${ }^{\text {a) }}$ \\
\hline $\operatorname{LVEF}(\%)$ & $65.2 \pm 2.3$ & $64.8 \pm 1.9$ & 0.875 \\
\hline Degree of MR & $2.3 \pm 0.7$ & $1.7 \pm 0.9$ & $<0.001$ \\
\hline $\mathrm{MR} \geq$ moderate & $13(23.6)$ & $8(14.5)$ & 0.279 \\
\hline Prolapse & $23(41.8)$ & $9(16.4)$ & 0.003 \\
\hline Annulus diameter (mm) & $32.7 \pm 8.9$ & $31.4 \pm 9.7$ & 0.099 \\
\hline Angle of AML on systole $\left({ }^{\circ}\right)$ & $2.4 \pm 9.4$ & $10.1 \pm 12.3$ & $<0.001$ \\
\hline Angle of PML on systole $\left({ }^{\circ}\right)$ & $12.1 \pm 22.2$ & $23.1 \pm 24.7$ & 0.003 \\
\hline Motion angle of AML $\left(^{\circ}\right)$ & $64.7 \pm 14.7$ & $59.7 \pm 13.8$ & 0.023 \\
\hline Motion angle of PML $\left(^{\circ}\right)$ & $52.9 \pm 33.4$ & $39.6 \pm 30.3$ & 0.001 \\
\hline AML length/annulus & \multicolumn{2}{|c|}{$0.8 \pm 0.1$} & \\
\hline PML length/annulus & \multicolumn{2}{|c|}{$0.5 \pm 0.2$} & \\
\hline
\end{tabular}

Values are presented as mean \pm standard deviation or number (\%).

LVEF, left ventricular ejection fraction; MR, mitral regurgitation; $A M L$, anterior mitral leaflet; $P M L$, posterior mitral leaflet.

${ }^{\text {a) }}$ Paired t-test or McNemar test. 


\section{PRECISION AND FUTURE MIEDICINE \\ Ok Jeong Lee, et al.}

the improvement in prolapse after surgery.

Significant postoperative changes in patients with a postoperative MR grade $\geq 3$ are shown in Table 3. Even though MR in most of our patients was less than moderate after surgery, eight patients (14.5\%) still had MR grade $\geq 3$. In all patients except two, the MR increased to grade $\geq 3$ after operation whereas MR didn't changed in two patients. Patients with a postoperative MR grade $<3$ showed significant im- provement in MVP, whereas there were no changes in MVP in patients with postoperative MR grade $\geq 3$. Motion angles of PML were significantly smaller in patients with postoperative MR grade $<3$ (Table 3). There were no significant changes in the motion angles of either leaflet in either patient group.

In terms of the effect of operative type on postoperative $M R$, patients with postoperative MR grade $<3$ underwent more chordae repairs but fewer leaflet repairs than the other

Table 2. Comparison between groups based on preoperative MR

\begin{tabular}{lccc}
\hline Variable & Preop. MR grade $<3$ & Preop. MR grade $\geq 3$ & P-value \\
\hline Number & 42 & 13 & 0.170 \\
Age at operation (yr) & $30.5 \pm 20.8$ & $39.8 \pm 21.9$ & 0.093 \\
Prolapse & $15(35.7)$ & $8(61.5)$ & 0.081 \\
Annulus diameter $(\mathrm{mm})$ & $31.5 \pm 8.7$ & $36.4 \pm 8.7$ & 0.178 \\
Angle of AML on systole $\left(^{\circ}\right)$ & $3.4 \pm 9.7$ & $-0.7 \pm 7.9$ & 0.498 \\
Angle of PML on systole $\left(^{\circ}\right)$ & $13.2 \pm 22.1$ & $8.4 \pm 22.9$ & 0.881 \\
Motion angle of AML $\left(^{\circ}\right)$ & $64.6 \pm 13.7$ & $65.3 \pm 18.2$ & 0.777 \\
Motion angle of PML $\left(^{\circ}\right)$ & $53.6 \pm 32.0$ & $50.5 \pm 39.1$ & 0.1 \\
AML length/annulus & $0.8 \pm 0.2$ & $0.7 \pm 0.1$ & 0.938 \\
PML length/annulus & $0.5 \pm 0.2$ & $0.5 \pm 0.2$ & \\
\hline
\end{tabular}

Values are presented as mean \pm standard deviation or number (\%).

MR, mitral regurgitation; Preop., preoperative; AML, anterior mitral leaflet; PML, posterior mitral leaflet.

Table 3. Comparison of changes after operation and variables associated with the operation between groups based on postoperative MR

\begin{tabular}{|c|c|c|c|c|c|c|}
\hline \multirow{2}{*}{ Variable } & \multicolumn{3}{|c|}{ Postop. MR grade $<3(n=47)$} & \multicolumn{3}{|c|}{ Postop. MR grade $\geq 3(n=8)$} \\
\hline & Preop. & Postop. & P-value & Preop. & Postop. & P-value \\
\hline Age at operation (yr) & \multicolumn{2}{|c|}{$28.0 \pm 18.9$} & & \multicolumn{2}{|c|}{$60.3 \pm 10.1$} & $<0.001$ \\
\hline Months to final echocardiography & \multicolumn{2}{|c|}{$36.0 \pm 38.7$} & & \multicolumn{2}{|c|}{$38.1 \pm 41.6$} & 0.895 \\
\hline Degree of MR & $2.3 \pm 0.7$ & $1.4 \pm 0.6$ & $<0.001$ & $2.5 \pm 0.7$ & $3.3 \pm 0.5$ & 0.330 \\
\hline Prolapse & $21(44.7)$ & $8(17.0)$ & 0.002 & $2(25.0)$ & $1(12.5)$ & 1.000 \\
\hline Annulus diameter (mm) & $30.6 \pm 7.7$ & $28.9 \pm 7.6$ & 0.071 & $44.6 \pm 4.4$ & $46.0 \pm 8.1$ & 0.779 \\
\hline Angle of AML on systole $\left(^{\circ}\right)$ & $1.9 \pm 9.5$ & $10.3 \pm 13.1$ & $<0.001$ & $5.0 \pm 9.3$ & $8.8 \pm 6.3$ & 0.327 \\
\hline Angle of PML on systole $\left(^{\circ}\right)$ & $11.3 \pm 23.8$ & $25.1 \pm 26.0$ & $<0.001$ & $16.9 \pm 7.3$ & $11.1 \pm 9.9$ & 0.263 \\
\hline Motion angle of $\mathrm{AML}\left({ }^{\circ}\right)$ & $64.5 \pm 15.2$ & $59.9 \pm 32.0$ & 0.086 & $66.3 \pm 12.2$ & $58.6 \pm 10.1$ & 0.208 \\
\hline Motion angle of $\mathrm{PML}\left({ }^{\circ}\right)$ & $58.8 \pm 32.0$ & $41.7 \pm 31.5$ & $<0.001$ & $19.5 \pm 19.5$ & $27.1 \pm 18.1$ & 0.327 \\
\hline LVEF (\%) & $63.4 \pm 4.9$ & $65.2 \pm 2.6$ & 0.745 & $65.2 \pm 3.2$ & $64.8 \pm 2.2$ & 0.847 \\
\hline Annuloplasty & \multicolumn{2}{|c|}{$42(89.4)$} & & \multicolumn{2}{|c|}{$7(87.5)$} & 1.000 \\
\hline Chordae repair & \multicolumn{2}{|c|}{$33(70.2)$} & & \multicolumn{2}{|c|}{$2(25.0)$} & 0.021 \\
\hline Leaflet repair & \multicolumn{2}{|c|}{$9(19.1)$} & & \multicolumn{2}{|c|}{$5(62.5)$} & 0.020 \\
\hline MAZE operation & \multicolumn{2}{|c|}{$10(21.3)$} & & \multicolumn{2}{|c|}{$7(87.5)$} & 0.001 \\
\hline
\end{tabular}

Values are presented as mean \pm standard deviation or number (\%).

MR, mitral regurgitation; Postop., postoperative; Preop., preoperative; AML, anterior mitral leaflet; PML, posterior mitral leaflet; LVEF, left ventricular ejection fraction. 
patients. However, there was no difference in the effect of annuloplasty on postoperative MR between the two groups. Age at operation was older (28.0 \pm 18.9 years vs. $60.3 \pm 10.1$ years) and more MAZE operations were performed in patients with a postoperative MR grade $\geq 3(21.3 \%$ vs. $87.5 \%)$ (Table 3).

\section{DISCUSSION}

We observed improvement in MR after concomitant surgery and ASD closure in most patients. Patients with postoperative MR grade $<3$ underwent chordae repair more frequently those with a postoperative MR grade $\geq 3$, whereas annuloplasty and leaflet repair were not frequently performed in patients with postoperative MR grade $<3$. MV motion was significantly altered by surgery in patients with postoperative MR grade $<3$. In addition, MAZE operations were performed more frequently in patients with MR grade $\geq 3$ and older patients.

Toyono et al. [6] suggested that MVP made a significant contribution to MR in ASD patients, and that isolated ASD closure resulted in a major improvement in MR. One study reported the disappearance of MVP in more than $30 \%$ of patients after ASD closure [7]. However, the other $70 \%$ of patients showed a significant improvement in MR after ASD closure only. Therefore, surgical repair of MVP should be considered concomitantly with ASD closure in those patients. Nishimura et al. [15] proposed aggravating factors for MR after isolated ASD closure such as thickened AML, shortened PML, LA dilatation, and perioperative atrial fibrillation. However, surgical indications for MR repair in patients with ASD and MR are not clear.

In our study, we measured angles of both mitral leaflets on systole and defined MVP as a negative angle value. Even though the motion of each mitral leaflet might be the secondary effect of volumetric changes, the analysis of the motions of each mitral leaflet could make the mechanisms of MR be clearer. The mobility and degree of prolapse of each leaflet could contribute MR independently or jointly before and after operation. MVP occurred in $41.8 \%$ of our patients, which is a higher percentage than reported previously (35\%) [6]. However, there might have been selection bias in our study because we evaluated patients who had undergone surgical MR repair. There were not significant differences of the angles of both leaflets on systole between patients with preoperative $M R \geq 3$ and preoperative $M R<3$ but those angles were smaller in patients with a postoperative MR grade $\geq 3$ than those with a postoperative MR grade $<3$. Further- more, not only MVP, but also the angle of the leaflet on systole improved after surgical repair. This improvement after surgery was more marked in patients with postoperative MR grade $<3$ than other patients. Therefore, we hypothesize that a good surgical MR repair outcome in patients with ASD is dependent on changes in the angles of both leaflets on systole. The motion angles of both leaflets are a secondary effect dependent on the angle of the leaflet on systole or diastole, but the motion of the angle, especially PML, showed a significant change after surgery. A few studies reported involvement of AML in more than half of ASD patients with MVP $[3,4,7]$, and that it disappeared after ASD closure. We found a significant change in the motion angle of PML, but we assumed that this was a consequence of changes in both leaflets, not only one leaflet. We recommend that attention should be given to PML in MR repair.

Patients with postoperative MR grade $<3$ were younger than those with postoperative MR grade $\geq 3$. Park et al. [9] reported that old age was a risk factor of MR after ASD closure even though their patients did not undergo MR repair. MV changes in ASD could be related to degeneration and volume overload. Geometrical changes of the right ventricle affect left ventricular change and MV, so that MR with MV changes occurs gradually before repair. We could not differentiate the cause of MR because we did not have any data of serial changes in MR before surgery in our patients. Therefore early operation is beneficial in terms not only of pulmonary hypertension, but also MR.

Our study showed that chordae repair was effective at reducing MR. The technique we used most commonly for chordae repair was chordae shortening, which could influence the angle of the leaflet on systole. Annuloplasty was performed extensively in our series of patients, but it alone did not prove to be effective. Interestingly, leaflet repair was performed more frequently in patients with postoperative MR grade $\geq 3$ versus those with postoperative MR grade $<3$, and we were not able to determine the effect of leaflet repair on postoperative MR in our patients. Chordae repair could have been responsible for significant changes in the angles of both leaflets on systole and the motion angle of leaflets.

We demonstrated that MAZE operations were more frequently performed in patients with postoperative MR grade $\geq 3$, consistent with previous observations $[9,10,15]$. Chronic atrial fibrillation can cause poor left ventricular function and dilatation that might induce MVP and MR. Other researchers have suggested that atrial fibrillation could cause left atrial distension and mitral annular dilatation, resulting in dis- 
placement of the posterior annulus and PML retraction with AML prolapse [16,17]. Therefore, patients who undergo the MAZE operation concomitant with ASD closure should be monitored closely for MR after surgery.

This study had several limitations. First, it was a retrospective study. Surgical indications and surgical procedures for MR were determined not based on universal criteria, but on individualized criteria. Quantification of MR was not performed because our study included old data. The interval of the last echocardiography from operation was variable because no follow-up protocol existed. Even though measurements were performed by a single person at one time, substantial errors could exist due to intra-personal variation and the variable qualities of the images. The small number of patients enrolled might have limited our ability to perform well-powered statistical analyses. There was also no control group in our study, such as a group comprising patients with ASD and MR who underwent isolated ASD closure. We were not sure about the effect of angles of both mitral leaflets on $M R$, so that it was not possible to suggest any goals of MV leaflets after MV surgery. Furthermore, when we analyzed the effect of surgical procedure on postoperative MR, we did not consider the combined effects and qualities of each procedure. Therefore well designed study with enough number of patients comprising MV surgery and control is mandatory.

In conclusion, outcomes of surgical MR repair performed concomitant with ASD closure were satisfactory. Not only prolapse, but also the angles of both leaflets on systole and the motion angle of PML were significantly improved in patients with postoperative MR grade $<3$. In terms of surgical procedure, chordae repair yielded better outcomes than other techniques, while older age at operation and MAZE operation yielded poorer outcomes.

\section{CONFLICTS OF INTEREST}

No potential conflict of interest relevant to this article was reported.

\section{ORCID}

Ok Jeong Lee

Jinyoung Song

June Huh

I-Seok Kang

Ji-Hyuk Yang

Tae-Gook Jun https://orcid.org/0000-0001-9234-1424

https://orcid.org/0000-0002-9660-5949

https://orcid.org/0000-0003-4523-9632

https://orcid.org/0000-0003-4264-0826 https://orcid.org/0000-0002-7349-6778 https://orcid.org/0000-0002-5161-4665

\section{REFERENCES}

1. Murphy JG, Gersh BJ, McGoon MD, Mair DD, Porter CJ, IIstrup DM, et al. Long-term outcome after surgical repair of isolated atrial septal defect. Follow-up at 27 to 32 years. N Engl J Med 1990;323:1645-50.

2. Campbell M. Natural history of atrial septal defect. $\mathrm{Br}$ Heart J 1970;32:820-6.

3. Joy J, Kartha CC, Balakrishnan KG. Structural basis for mitral valve dysfunction associated with ostium secundum atrial septal defects. Cardiology 1993;82:409-14.

4. Suchon E, Podolec P, Plazak W, Tomkiewicz-Pajak L, Pieculewicz M, Mura A, et al. Mitral valve prolapse associated with ostium secundum atrial septal defect: a functional disorder. Acta Cardiol 2004;59:237-8.

5. Angel J, Soler-Soler J, Garcia del Castillo H, Anivarro I, Batlle-Diaz J. The role of reduced left ventricular enddiastolic volume in the apparently high prevalence of mitral valve prolapse in atrial septal defect. Eur J Cardiol 1980; 11:341-55.

6. Toyono M, Pettersson GB, Matsumura Y, Wada N, Fukuda $\mathrm{S}$, Yamano $\mathrm{T}$, et al. Preoperative and postoperative mitral valve prolapse and regurgitation in adult patients with secundum atrial septal defects. Echocardiography 2008; 25:1086-93.

7. Nagata S, Nimura Y, Sakakibara H, Beppu S, Park YD, Kawazoe K, et al. Mitral valve lesion associated with secundum atrial septal defect. Analysis by real time two dimensional echocardiography. Br Heart J 1983;49:51-8.

8. Takaya Y, Kijima Y, Akagi T, Nakagawa K, Oe H, Taniguchi M, et al. Fate of mitral regurgitation after transcatheter closure of atrial septal defect in adults. Am J Cardiol 2015; 116:458-62.

9. Park JJ, Lee SC, Kim JB, Seo DJ, Song JM, Yun SC, et al. Deterioration of mitral valve competence after the repair of atrial septal defect in adults. Ann Thorac Surg 2011;92: 1629-33.

10. Yoshida S, Numata S, Tsutsumi Y, Monta O, Yamazaki S, $\mathrm{Seo} \mathrm{H}$, et al. Mitral valve regurgitation after atrial septal defect repair in adults. J Heart Valve Dis 2014;23:310-5.

11. Nakagawa K, Akagi T, Taniguchi M, Kijima Y, Goto K, Kusano KF, et al. Transcatheter closure of atrial septal defect in a geriatric population. Catheter Cardiovasc Interv 2012; 80:84-90.

12. Wilson NJ, Smith J, Prommete B, O'Donnell C, Gentles TL, Ruygrok PN. Transcatheter closure of secundum atrial septal defects with the amplatzer septal occluder in 
adults and children-follow-up closure rates, degree of mitral regurgitation and evolution of arrhythmias. Heart Lung Circ 2008;17:318-24.

13. Hiraishi M, Tanaka H, Motoji Y, Sawa T, Tsuji T, Miyoshi T, et al. Impact of right ventricular geometry on mitral regurgitation after transcatheter closure of atrial septal defect. Int Heart J 2015;56:516-21.

14. Lang RM, Bierig M, Devereux RB, Flachskampf FA, Foster E, Pellikka PA, et al. Recommendations for chamber quantification: a report from the American Society of Echocardiography's Guidelines and Standards Committee and the Chamber Quantification Writing Group, developed in conjunction with the European Association of Echocardi- ography, a branch of the European Society of Cardiology. J Am Soc Echocardiogr 2005;18:1440-63.

15. Nishimura S, Izumi C, Amano M, Miyake M, Tamura T, Kondo $\mathrm{H}$, et al. Incidence and predictors of aggravation of mitral regurgitation after atrial septal defect closure. Ann Thorac Surg 2017;104:205-10.

16. Silbiger JJ. Novel pathogenetic mechanisms and structural adaptations in ischemic mitral regurgitation. J Am Soc Echocardiogr 2013;26:1107-17.

17. Takahashi Y, Abe Y, Sasaki Y, Bito Y, Morisaki A, Nishimura $S$, et al. Mitral valve repair for atrial functional mitral regurgitation in patients with chronic atrial fibrillation. Interact Cardiovasc Thorac Surg 2015;21:163-8. 